\title{
Ab initio energetics of phosphorus impurity in subsurface regions of hydrogenated diamond surfaces *
}

\author{
Takehide Miyazaki ${ }^{\dagger}$ \\ Research Institute for Computational Sciences, National Institute of Advanced Industrial Science and Technology (AIST), \\ AIST Tsukuba Central 2, Tsukuba 305-8568, Japan
}

Hiromitsu Kato, Hideyo Okushi, and Satoshi Yamasaki

Diamond Research Center, AIST, AIST Tsukuba Central 2, Tsukuba 305-8568, Japan

(Received 13 November 2005; Accepted 28 November 2005; Published 26 January 2006)

\begin{abstract}
We present first-principles energetics of a phosphorus $(\mathrm{P})$ impurity atom substitutionally doped in the vicinity of the $\mathrm{C}(001)-2 \times 1: \mathrm{H}$ and $\mathrm{C}(111)-1 \times 1: \mathrm{H}$ surfaces. We find that locating the $\mathrm{P}$ atom within topmost four $\mathrm{C}$-atomic layers of the $\mathrm{C}(001)-2 \times 1: \mathrm{H}$ surface substantially lowers the formation energy relative to that for doping in the bulk, where the energy lowering $\left(\Delta E_{\text {form }}\right)$ is up to $\sim 4 \mathrm{eV}$. For $\mathrm{C}(111)-1 \times 1: \mathrm{H}$, the $\Delta E_{\text {form }}$ is up to $\sim 2.5 \mathrm{eV}$. Thus we expect that the doped $\mathrm{P}$ atoms may have a strong tendency of segregation close to these surfaces. We will also discuss an implication of our result in the context of a recent experiment of P-doping of the (001) and (111) oriented diamond films [H. Kato, S. Yamasaki and H. Okushi, Appl. Phys. Lett. 86, 222111 (2005)].
\end{abstract}

[DOI: $10.1380 /$ ejssnt.2006.124]

Keywords: Density functional calculations; Surface energy; Diamond; Phosphorus

\section{INTRODUCTION}

The electronic and chemical properties of diamond semiconductors are superior to those of conventional semiconductors such as silicon and germanium because of its large energy band gap, $\sim 5.5 \mathrm{eV}$. This fact has motivated numerous studies toward potential applications of diamond semiconductors to power devices, bio-chemical sensors, ultra-violet light sources, and so on. For these applications to be successful in industrial fields, it is at least required to establish technology to control the populations of electrons and holes by doping the materials with impurity atoms to obtain n-type [with phosphorus $(\mathrm{P})$ ] and p-type (with boron) electrical conductivities, respectively.

However, P-doping of diamond has turned out to be difficult. In most cases, P-doping of diamond is performed during growth of the diamond crystal itself using chemical vapor deposition (CVD). One of currently serious difficulties is that the efficiency of incorporation of $\mathrm{P}$ atoms in crystal during CVD growth is sensitive to the orientation of the crystal surface: P-doping of diamond has been successful only for the (111)-oriented samples[1]. It was not until very recent that doping of $\mathrm{P}$ atoms in the (001)oriented diamond films during microwave CVD growth was achieved $[2]$. The ratio of $[\mathrm{P}] /[\mathrm{C}]$ in the diamond film relative to $\left[\mathrm{PH}_{3}\right] /\left[\mathrm{CH}_{4}\right]$ in the $\mathrm{CVD}$ gas phase is about $0.02 \%$, which is two orders of magnitude smaller than that in the (111) films, although the absolute P-content was made roughly the same as the counterpart for the (111) films. Since efficient P-doping of (001) diamond films is very important in application of diamond semiconductors to the above mentioned devices, it is strongly desired to improve the P-doping efficiency in the (001) samples. Apart from the fact that P-doping of diamond

\footnotetext{
* This paper was presented at International Symposium on Surface Science and Nanotechnology (ISSS-4), Saitama, Japan, 14-17 November, 2005.

†Corresponding author: takehide.miyazaki@aist.go.jp
}

during CVD growth should undergo very complicated processes, the experiment suggests at least that the surface itself may play a key role in $\mathrm{P}$-doping of diamond.

On the theoretical side, great efforts have been made to understand mechanisms of doping of Si during CVD[3] and molecular beam epitaxy[4]. The doping mechanisms may be summarized in two regimes, dopants on the surface [regime $(\mathrm{A})]$ and in the crystal [regime (B)]: (A) The impurity atoms are attached to the terrace and then trapped at the steps or kinks via surface diffusion and finally embedded in the crystal film due to subsequent attachment of Si atoms. (B) The impurity atoms are distributed into the crystal following their diffusivity (distinct from surface diffusion) and the potential energy surface.

The goal of this study is that we understand based on first-principles calculation the effect of the surface structure on the process of P-doping of diamond. As a first step toward this goal, we focus our viewpoint on the regime (B): the impurity in the near-surface positions. Since creation of the surface often affects the atomic and electronic structures of the subsurface region, the difference in the surface geometries should be reflected in the P-doping energetics in the near-surface positions of the (001) and (111) diamond surfaces. Our viewpoint is also inspired by the fact that in case of doping of silicon the population of impurity atoms can become much higher near surfaces than the counterpart in bulk regions. One generic example of such situations is the enhanced solubility of carbon (C) atoms near $\mathrm{Si}(001)-2 \times 1[5]$.

In concrete, we calculate the total energies of optimized structures for mono-hydrogenated $\mathrm{C}(001)$ and $\mathrm{C}(111)$ surfaces [denoted as $\mathrm{C}(001)-2 \times 1: \mathrm{H}$ and $\mathrm{C}(111)-1 \times 1: \mathrm{H}$, respectively], where a $\mathrm{P}$ atom substitutes for the $\mathrm{C}$ sites in the atomic layers near each surface. We repeat the totalenergy calculations as a function of the position of the $\mathrm{P}$ atom which ranges from the first to fifth (fourth) surface layers of $\mathrm{C}(001)-2 \times 1: \mathrm{H}[\mathrm{C}(111)-1 \times 1: \mathrm{H}]$. We find that the energies of the $\mathrm{P}$ atom near the surfaces are much lower than that for $\mathrm{P}$ in the bulk. This lowering in the energy, $\Delta E_{\text {form }}$, defined in eq.(1), is estimated up to $\sim 4 \mathrm{eV}$ and 
up to $\sim 2.5 \mathrm{eV}$ for $\mathrm{C}(001)-2 \times 1: \mathrm{H}$ and $\mathrm{C}(111)-1 \times 1: \mathrm{H}$, respectively. Comparing to the $\Delta E_{\text {form }}$ values in the case of a substitutional $\mathrm{C}$ atom near $\mathrm{Si}(001)-2 \times 1$ which are $\leq \sim 0.8 \mathrm{eV}[5]$, we conclude that the doped $\mathrm{P}$ atoms are very likely to be segregated near hydrogenated diamond surfaces. We also expect that the tendency of segregation should be stronger for $\mathrm{C}(001)-2 \times 1$ : $\mathrm{H}$ than $\mathrm{C}(111)-1 \times 1$ : $\mathrm{H}$. We will further discuss an implication of our result in light of the above mentioned experimental finding.

\section{METHOD OF CALCULATION}

We adopt an $a b$ initio theoretical method to study energetics of substitutional $\mathrm{P}$ impurity near the $\mathrm{C}(001)-2 \times 1: \mathrm{H}$ and $\mathrm{C}(111)-1 \times 1: \mathrm{H}$ surfaces. The electronic structures are calculated based on density-functional theory $[6,7]$ within generalized gradient approximation $[8]$ to the exchangecorrelation energies. The $\mathrm{C}(001)-2 \times 1: \mathrm{H}$ and $\mathrm{C}(111)$ $1 \times 1: H$ surface structures are modeled in supercells with the $4 \times 4$ surface periodicity. In the surface-normal direction, the size of a supercell and the thickness of a slab located within the supercell are $24 \mathrm{C}$ layers long and $\sim 16$ C layers thick (30 C layers long and $\sim 18 \mathrm{C}$ layers thick) for $\mathrm{C}(001)-2 \times 1: \mathrm{H}[\mathrm{C}(111)-1 \times 1: \mathrm{H}]$, respectively (see a note [9] for a precise description of the supercells). All slab geometries we consider are configured with inversion symmetry. The integration over the whole first Brillouin zone is performed with the $2 \times 2 \times 1 \mathbf{k}$ points. The wave functions are expanded in plane-wave basis set up to $25 \mathrm{Ry}$. The ultrasoft[10, 11] (for $\mathrm{C}$ and $\mathrm{H}$ ) and norm-conserving[12] (for P) pseudopotentials are used to eliminate the core states. The augmentation functions are expanded in plane waves up to 400 Ry. The self-consistent field iterations are continued until the total energy converges to $\sim 3 \times 10^{-5}$ $\mathrm{eV} /$ atom. The atomic positions of all atoms in supercell are relaxed until the residual forces are less than $\sim 0.052$ $\mathrm{eV} / \AA$.

\section{RESULTS}

In order to study the energetics of $\mathrm{P}$-doping in the subsurface regions of the $\mathrm{C}(001)-2 \times 1: \mathrm{H}$ and $\mathrm{C}(111)-1 \times 1: \mathrm{H}$ surfaces, we calculate the difference in the formation energy of a $\mathrm{P}$ near each surface from that in bulk. Let us denote this difference by $\Delta E_{\text {form }}$, defined as[13]

$\Delta E_{\mathrm{form}}=\left\{E_{\text {tot }}^{\text {bulk }}(\mathrm{P})+E_{\text {tot }}^{\text {surface }}(0)\right\}-\left\{E_{\text {tot }}^{\text {bulk }}(0)+E_{\text {tot }}^{\text {surface }}(\mathrm{P})\right\}$,

where $E_{\text {tot }}^{\text {bulk }}(\mathrm{P})\left[E_{\text {tot }}^{\text {bulk }}(0)\right]$ and $E_{\text {tot }}^{\text {surface }}(\mathrm{P})\left[E_{\text {tot }}^{\text {surface }}(0)\right]$ are the total energies of bulk diamond and either of the two surfaces with (without) a substitutional $\mathrm{P}$, both at the neutral charge state, respectively. If $\Delta E_{\mathrm{form}}>0$, then the substitutional $\mathrm{P}$ favors to be near surfaces rather than in a bulk crystal. In other words, our target quantity $\Delta E_{\text {form }}$ is the "binding energy" of phosphorus-surface "complexes" and we aim at investigation of the stability of those "complexes". We calculated $E_{\mathrm{tot}}^{\text {bulk }}(\mathrm{P})$ using the (001) and (111) bulk supercells defined in [9], which are fulfilled with $447 \mathrm{C}$ plus one $\mathrm{P}$ and $479 \mathrm{C}$ plus one $\mathrm{P}$ atoms, respectively. To make a comparison of $\Delta E_{\mathrm{form}}$

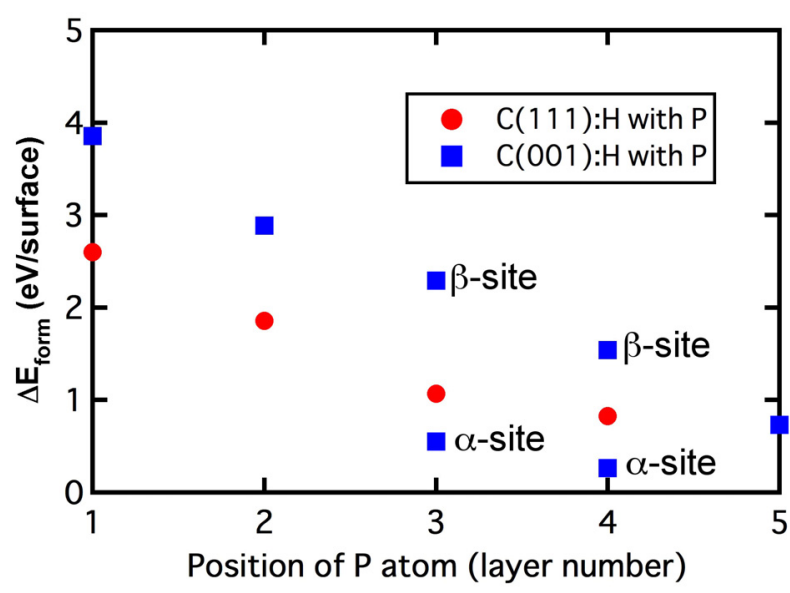

FIG. 1: Formation-energy gain $\left(\Delta E_{\text {form }}\right.$, see text for definition) as a function of the position of a substitutional $\mathrm{P}$ atom near the $\mathrm{C}(001)-2 \times 1$ :H and $\mathrm{C}(111)-1 \times 1$ :H surfaces. The number in the horizontal axis is the depth of $\mathrm{C}$ layers from the topmost layers of each surface. For example, the layer number 1 means the first $\mathrm{C}$ layer of each surface.

for the two surfaces on the same footing, we corrected the spurious difference in $E_{\mathrm{tot}}^{\mathrm{bulk}}(\mathrm{P})$ calculated with the (001) and (111) bulk supercells, $\sim 0.34 \mathrm{eV}$ per $\mathrm{P}$ atom. We did not include the contribution of zero-point energy of $\mathrm{H}$ atoms to our energetics.

In Fig.1, we show $\Delta E_{\text {form }}$ for the $\mathrm{C}(001)-2 \times 1: \mathrm{H}$ and $\mathrm{C}(111)-1 \times 1: \mathrm{H}$ surfaces. As is clear from the figure, a substitutional $\mathrm{P}$ atom favors to be located in the first $\mathrm{C}$ layer of the both surfaces. The reduction of the formation energy propagates over at least $4 \sim 5 \mathrm{C}$ layers of both surfaces. The $\Delta E_{\text {form }}$ values are larger for $\mathrm{C}(001)$ $2 \times 1$ :H (except those at the $\alpha$-sites, see below) than those for $\mathrm{C}(111)-1 \times 1: \mathrm{H}$ especially at the first $\mathrm{C}$ layer, $\sim 4 \mathrm{eV}$ for the former and $\sim 2.5 \mathrm{eV}$ for the latter. Because of a large size mismatch between $\mathrm{P}$ and $\mathrm{C}$ atoms, locating a

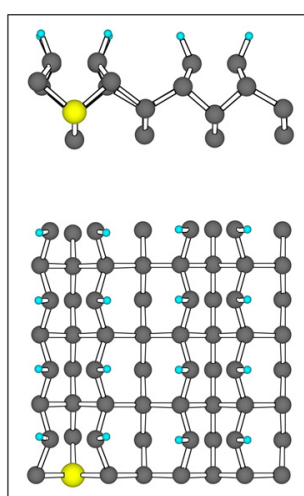

(a)

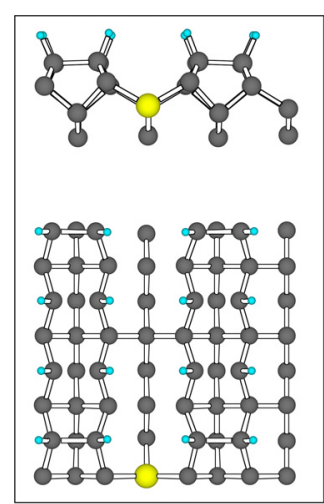

(b)

FIG. 2: Ball-stick models of optimized structures of the $\mathrm{C}(001)-2 \times 1$ : H surface with a $\mathrm{P}$ atom doped in the third $\mathrm{C}$ layer. Grey, yellow and blue balls illustrate C, P and $\mathrm{H}$ atoms, respectively. The sticks represent bonds between atoms, which are drawn if the C-C, C-P and C-H distances are shorter than $1.6 \AA, 2.0 \AA$ and $1.2 \AA$, respectively. (a) The $\mathrm{P}$ atom at the $\alpha$ site. (b) The $\mathrm{P}$ atom at the $\beta$-site. In each panel, only four $\mathrm{C}$ layers near the surface are drawn for clarity. 
$\mathrm{P}$ atom in the bulk region of diamond crystal is not energetically preferential. In contrast, the energy loss due to P-doping can be efficiently reduced by virtue of a greater lattice flexibility of the near-surface regions. The overall feature of the $\Delta E_{\text {form }}$ as a function of the depth of the $\mathrm{P}$-atom location found for both diamond surfaces is quite similar to what has been reported for a substitutional $\mathrm{C}$ near $\operatorname{Si}(001)-2 \times 1[5]$.

Let us describe the result for $\mathrm{P}$ in $\mathrm{C}(001)-2 \times 1: \mathrm{H}$ in more detail. Due to the $2 \times 1$ reconstruction of this surface, its third and fourth layers have two non-equivalent sites for substitution with $\mathrm{P}$, the one below the surface dimer rows [the $\alpha$-site, Fig.2(a)], and the other between two adjacent surface dimer rows [the $\beta$-site, Fig.2(b)]. The $\mathrm{P}$ atom in both the third and fourth layers is very much likely to sit in the $\beta$-sites, for which the $\Delta E_{\text {form }}$ are $\sim 1.7 \mathrm{eV}$ (in the third layer) and $\sim 1.3 \mathrm{eV}$ (in the fourth layer) larger than the respective counterparts for the $\alpha$-sites. As has been known for $\mathrm{Si}(001)-2 \times 1[5]$, the $\alpha$-site is under compressive stress but the $\beta$-site is under expansive stress. The same should apply to $\mathrm{C}(001)-2 \times 1: \mathrm{H}$. Since a $\mathrm{P}$ atom is larger in size than a $\mathrm{C}$ atom, putting the $\mathrm{P}$ atom at a $\beta$-site is very favorable for relaxing the expansive stress at this site. Our present result is similar to the prediction made in the case of $\mathrm{Si}(001)-2 \times 1$ that when the substitutional atom is too large rather than too small, as for $\mathrm{Sb}$ in $\mathrm{Si}$, it will prefer the $\beta$-sites rather than the $\alpha$-sites[5]. For a substitutional $\mathrm{C}$ near $\mathrm{Si}(001)-2 \times 1[5]$, the $\mathrm{C}$ atom favors the $\alpha$-site, because its size is smaller than $\mathrm{Si}$, which may relax the compressive stress at the $\alpha$-site.

However, there are also some important differences in doping energeitcs between the present P-doping near diamond surfaces and C-doping near $\mathrm{Si}(001)-2 \times 1[5]$. (i) The $\Delta E_{\text {form }}$ values for $\mathrm{P}$-doping of both $\mathrm{C}(001)-2 \times 1: \mathrm{H}$ and $\mathrm{C}(111)-1 \times 1$ are much larger (up to $\sim 4 \mathrm{eV}$ ) than the counterpart for C-doping near $\mathrm{Si}(001)-2 \times 1$, which is $0.8 \mathrm{eV}$ at most. This stems from the fact that the chemical bonds of diamond crystal is much stronger than that of silicon crystal. (ii) Locating a $\mathrm{P}$ atom at the $\alpha$-sites of $\mathrm{C}(001)$ $2 \times 1$ : $\mathrm{H}$ gains the formation energy $(\sim 0.5 \mathrm{eV})$ relative to $\mathrm{P}$ in bulk, although this site is predicted unfavorable for too large impurity atoms for $\mathrm{Si}[5]$.

Judging from the results presented so far, we predict that doping of diamond surfaces with $\mathrm{P}$ atoms may have a stronger tendency to induce segregation of the $\mathrm{P}$ atoms at the surfaces than the C-doping near $\mathrm{Si}(001)-2 \times 1$.

Hereafter we note on the configurations of a $\mathrm{P}$ atom in the first $\mathrm{C}$ layer for the diamond surfaces studied here (Fig.3). The lowest-energy structure of $\mathrm{C}(111)-1 \times 1: \mathrm{H}$ has no $\mathrm{H}$ atom atop of the $\mathrm{P}$ atom [Fig.3(a)]. We found a local-minimum structure with an $\mathrm{H}$ atom being at the on-top position of the $\mathrm{P}$ atom (the $\mathrm{P}-\mathrm{H}$ distance being $\sim 2.06 \AA$ ), whose energy is $\sim 0.4 \mathrm{eV}$ per surface higher than that of the structure in Fig.3(a) plus an isolated $\mathrm{H}$ atom. Since a $\mathrm{P}$ atom has five valence electrons and the firstlayer $\mathrm{P}$ atom has three $\mathrm{P}-\mathrm{C}$ back bonds (the $\mathrm{P}-\mathrm{C}$ distance is $\sim 1.80 \AA$ ) with the $\mathrm{C}(111)$ surface, the remaining two valence electrons create a lone pair. Thus there is little necessity for hydrogenation of the first-layer $\mathrm{P}$ atom on $\mathrm{C}(111)-1 \times 1: \mathrm{H}$. The $\mathrm{C}-\mathrm{P}-\mathrm{C}$ angle is $\sim 96.7^{\circ}$, which is much smaller than the tetrahedral angle $\sim 109.5^{\circ}$ but slightly larger than the $\mathrm{H}-\mathrm{P}-\mathrm{H}$ angle $\sim 92.4^{\circ}$ of a $\mathrm{PH}_{3}$ molecule.

As for $\mathrm{C}(001)-2 \times 1: \mathrm{H}$, an $\mathrm{H}$ atom is bound to the

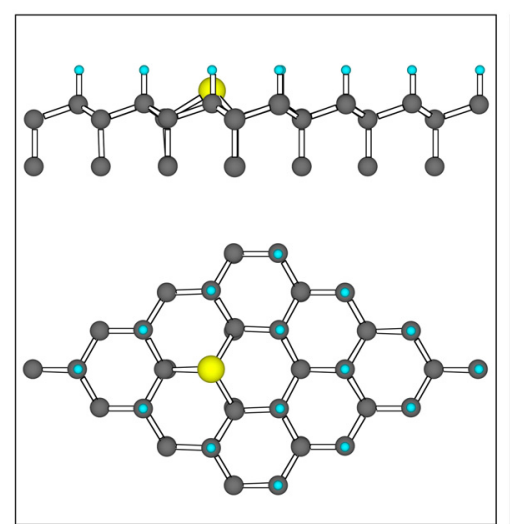

(a)

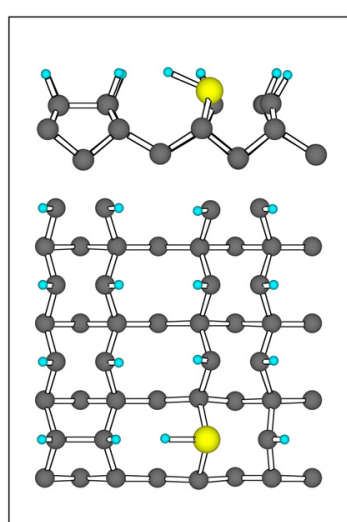

(b)
FIG. 3: Ball-stick models of optimized structures of the (a) $\mathrm{C}(111)-1 \times 1: \mathrm{H}$ and (b) $\mathrm{C}(001)-2 \times 1: \mathrm{H}$ surfaces with a $\mathrm{P}$ atom doped in the first $\mathrm{C}$ layer, respectively. The convention is the same as in Fig.2 except that the sticks between $\mathrm{P}$ and $\mathrm{H}$ atoms are drawn if the $\mathrm{P}-\mathrm{H}$ distance is shorter than $1.5 \AA$. In each panel, only three $\mathrm{C}$ layers near the surface are drawn for clarity.

first-layer $\mathrm{P}$ atom with the $\mathrm{P}-\mathrm{H}$ length being $\sim 1.48 \AA$ in our lowest-energy structure [Fig.3(b)]. The distance of the $\mathrm{P}-\mathrm{C}$ back bond is $\sim 1.84 \AA$ while for the first-layer P-C "dimer" the separation between the two atoms is $\sim 2.05 \AA$. The $\mathrm{P}-\mathrm{H}$ length $(\sim 1.48 \AA)$ is close to that of $\mathrm{PH}_{3}$ $(\sim 1.42 \AA)$. The H-P-C bond angle is $\sim 97.6^{\circ}$, also similar to the $\mathrm{C}-\mathrm{P}-\mathrm{C}$ angle $\left(\sim 96.7^{\circ}\right)$ of the first-layer $\mathrm{P}$ on $\mathrm{C}(111)-1 \times 1: \mathrm{H}$. If we isolate the $\mathrm{H}$ atom attached to $\mathrm{P}$ in Fig.3(b) in the vacuum, then the resultant total energy is $\sim 0.13 \mathrm{eV}$ per surface higher than that of the geometry in Fig.3(b). In this case, the distance between $\mathrm{P}$ and its nearest first-layer $\mathrm{C}$ is $\sim 1.93 \AA$. In short, local atomic configurations of the first-layer $\mathrm{P}$ atoms on $\mathrm{C}(111)-1 \times 1: \mathrm{H}$ and $\mathrm{C}(001)-2 \times 1: \mathrm{H}$ may be regarded similar to each other.

\section{DISCUSSION}

A reason for why $\Delta E_{\text {form }}$ is larger for $\mathrm{C}(001)-2 \times 1: \mathrm{H}$ than $\mathrm{C}(111)-1 \times 1: \mathrm{H}$ appears to originate from the difference in the stability for these surfaces without impurities: the former is less favorable to create than the latter. It has been shown[14] that the formation energy of $\mathrm{C}(001)$ $2 \times 1: \mathrm{H}$ is always higher than that of $\mathrm{C}(111)-1 \times 1: \mathrm{H}$ for hydrogen chemical potential $\left(\mu_{\mathrm{H}}\right)$ lower than a threshold $\left(\mu_{\mathrm{H}}^{0}\right)[15]$ over which the $\mathrm{CH}_{4}$ molecules could be spontaneously desorbed from the surface. The difference in the formation energies may be attributed to the different atomic structures of these surfaces.

Keeping the above in mind, we discuss a cause of the difference in the $\Delta E_{\text {form }}$ values of $\mathrm{P}$ doping for $\mathrm{C}(001)$ $2 \times 1: \mathrm{H}$ and $\mathrm{C}(111)-1 \times 1: \mathrm{H}$. Since the first-layer $\mathrm{C}$ atoms of $\mathrm{C}(111)-1 \times 1: \mathrm{H}$ is tetrahedrally coordinated due to $\mathrm{H}$ capping, replacing the surface $\mathrm{C}-\mathrm{H}$ unit by a $\mathrm{P}$ atom may not be very favorable, although the three coordination around the $\mathrm{P}$ atom at surface [Fig.3(b)] does indeed gain an energy relative to the tetrahedral $\mathrm{P}$ in the bulk. In 
contrast, in case of the $\mathrm{P}$-doping of $\mathrm{C}(001)-2 \times 1$ : $\mathrm{H}$, dimerization of the surface $\mathrm{C}$ atoms induces distortions in the subsurface region, as was explained in the result section. Replacement of a $\mathrm{C}$ atom of a surface dimer by a $\mathrm{P}$ atom appears to greatly relax the distortion, which thus substantially reduces the formation energy in comparison to that for a $\mathrm{P}$ in a bulk diamond crystal.

Next, we discuss an implication of the present result in the context of a recent experiment about P-doping of (001) and (111) diamond films during CVD growth[2]. Our current interest is in the reason for their success in the P-doping of the (001) films with the P-content quite similar to that of (111). Although we should clarify not only surface segregation but also many other aspects of the P-doping in order to give a complete answer to this question, let us try to make a qualitative speculation with a conceptual help of the existing theory in the regime (B) [4].

The authors of Ref.[2] have found that in order to obtain the same P-concentration $\left(\sim 10^{18} \mathrm{~cm}^{-3}\right)$ for these orientations, it is required to apply both higher $\left[\mathrm{CH}_{4}\right] /\left[\mathrm{H}_{2}\right]$ ratio $(0.1-1.0 \%)$ and $\left[\mathrm{PH}_{3}\right] /\left[\mathrm{CH}_{4}\right]$ ratio (1$10 \%)$ to the (001) films than those optimized for the (111) films, which are $0.05-0.1 \%$ and $100-1000 \mathrm{ppm}$, respectively. This result has been obtained setting the same substrate temperatures $T_{s}\left(850-950^{\circ} \mathrm{C}\right)$, gas pressures $p_{\text {gas }}\left(25-75\right.$ Torr) and microwave power $P_{\mathrm{MW}}$ $(750 \mathrm{~W})$ for these two orientations. The growth rates of the (001) and (111) films are similar, $~ 360$ $\mathrm{nm} / \mathrm{h}\left(T_{s}=900^{\circ} \mathrm{C},\left[\mathrm{CH}_{4}\right] /\left[\mathrm{H}_{2}\right]=0.05 \%,\left[\mathrm{PH}_{3}\right] /\left[\mathrm{CH}_{4}\right]=500\right.$ ppm, $\quad p_{\text {gas }}=25$ Torr, $\left.P_{\mathrm{MW}}=750 \mathrm{~W}\right)[16]$ and $\sim 250$ $\mathrm{nm} / \mathrm{h}\left(T_{s}=900^{\circ} \mathrm{C},\left[\mathrm{CH}_{4}\right] /\left[\mathrm{H}_{2}\right]=0.4 \%,\left[\mathrm{PH}_{3}\right] /\left[\mathrm{CH}_{4}\right]=5 \%\right.$, $p_{\text {gas }}=25$ Torr, $\left.P_{\mathrm{MW}}=750 \mathrm{~W}\right)[17]$, respectively. Growing on (001) samples with parameters optimized for (111) doping does not result in any detectable incorporation of $\mathrm{P}[2]$.

What we can learn from the above experiment is that the growth rate $(R)$ of the crystal and the rate of the change in the number of $\mathrm{P}$ atoms doped in the first $\mathrm{C}$ layer $(F)$ of the crystal surface appear to be very important parameters to affect the $\mathrm{P}$-incorporation in the diamond films. Of course, we do not mean that all the physics of P-doping of diamond should be governed by $R$ and $F$ only. We assume that $R$ and $F$ depend on the $\left[\mathrm{CH}_{4}\right] /\left[\mathrm{H}_{2}\right]$ and $\left[\mathrm{PH}_{3}\right] /\left[\mathrm{CH}_{4}\right]$ ratios in the CVD gas phase, respectively. At the end of this discussion section, we will comment on a possible dependence of $R$ and $F$ on each other. We introduce the specific time $(\tau)$ required to grow one atomic layer of the crystal film with the thickness $h$, $\tau=h / R$. We expect that $F$ may be determined by a balance between the fluxes of the dopants supplied to, and escaping from the surface.

In the regime $(\mathrm{B})$ of doping model, diffusion and surface segregation are main driving forces to determine distribution of the impurity atoms. Let us consider the film growth by one crystal layer, during which the $N_{s}(=F \tau)$ atoms are doped in the topmost layer. At any $N_{s}$, two dif- ferent cases of impurity distribution may occur, an equilibrium distribution for a large $\tau$ (a low $R$ and a small $F$ ) and a kinetically limited distribution for a small $\tau$ (a high $R$ and a large $F$ )[4]. In the equilibrium due to slow growth, the doped impurity atoms would be segregated in the near-surface region or otherwise diffused away in the crystal, depending on the $\Delta E_{\text {form }}$ values as a function of the position of the impurity atom. On the other hand, fast growth may lead to non-equilibrium distributions of the dopants if $\tau$ is taken to be shorter than the relaxation time for achievement of the equilibrium distribution. In the latter case, the impurity population near the crystal surface per atomic layer may be frozen around $F \tau$.

Turning to the experiment in Ref.[2], we speculate that the P-doping of (001) samples with the growth condition optimized for (111) doping led to a very slow growth of the (001) films. Due to the larger $\Delta E_{\text {form }}$ values than those for the (111) surface, the slow growth may cause segregation of the $\mathrm{P}$ atoms in the subsurface region. This may become one candidate of the reasons to explain why it was necessary to set the much higher $\left[\mathrm{CH}_{4}\right] /\left[\mathrm{H}_{2}\right]$ (to achieve a high $R$ ) and $\left[\mathrm{PH}_{3}\right] /\left[\mathrm{CH}_{4}\right]$ (to achieve a high $F$ ) ratios than the counterparts for the (111) doping, in order to dope the (001) samples at as high the P-content as that for the (111) samples. As for a possible cause of the low Pincorporation ratio in the (001) case, we may speculate at present that $F$ might depend on $\tau$ itself (or equivalently, the inverse of the growth rate $R$ ). If so, $F$ would be greatly reduced for large $R$ values due to increasing opportunity of arrival of the $\mathrm{C}$ species at the growth front.

\section{CONCLUSION}

In conlcusion, our ab initio calculation reveals a strong segregation tendency of the $\mathrm{P}$ atoms to the surface. We also find that the energetics of P-doping is sensitive to the surface orientation. Especially, the $\Delta E_{\text {form }}$ estimated for the $\mathrm{C}(001)-2 \times 1: \mathrm{H}$ and $\mathrm{C}(111)-1 \times 1$ surfaces are $\sim 4 \mathrm{eV}$ and $\sim 2.5 \mathrm{eV}$, respectively, when the $\mathrm{P}$ atom substitutes for the first-layer $\mathrm{C}$ atoms. The result may provide us with some insight into a reason for a recent success in the CVD P-doping of the (001)-oriented diamond films[2]. For constructing a more accurate understanding of the physics of P-doping of the (001) and (111) diamond films, experimental as well as theoretical elucidation of atomistic surface processes and diffusion mechanisms of $\mathrm{P}$ atoms in the subsurface regions together with energetics of various defects near surfaces should be performed, which are now under way.

\section{Acknowledgments}

One of the authors (T.M.) thanks Dr. S.-G. Ri, Dr. M. Ogura and Dr. D. Takeuchi for their useful comments.
[1] S. Koizumi, M. Kamo, Y. Sato, H. Ozaki, and T. Inuzuka, Appl. Phys. Lett. 71, 1065 (1997).
[2] H. Kato, S. Yamasaki, and H. Okushi, Appl. Phys. Lett. 86, 222111 (2005). 
[3] R. Reif and R. W. Dutton, J. Electrochem. Soc. 128, 909 (1981).

[4] H. Jorke, Surf. Sci. 193, 569 (1988).

[5] J. Tersoff, Phys. Rev. Lett. 74, 5080 (1995).

[6] P. Hohenberg and W. Kohn, Phys. Rev. 136, B864 (1964).

[7] W. Kohn and L. J. Sham, Phys. Rev. 140, A1133 (1965).

[8] J. P. Perdew, K. Burke, and M. Ernzerhof, Phys. Rev. Lett. 77, 3865 (1996).

[9] The translation vectors of the supercells representing the $\mathrm{C}(001)$ and $\mathrm{C}(111)$ surfaces are

$A=\left(\begin{array}{ccc}N \eta & 0 & 0 \\ 0 & N \eta & 0 \\ 0 & 0 & M c\end{array}\right) \quad$ and $\quad A^{\prime}=\left(\begin{array}{ccc}\frac{\sqrt{3}}{2} N \eta & \frac{\sqrt{3}}{2} N \eta & 0 \\ -\frac{1}{2} N \eta & \frac{1}{2} N \eta & 0 \\ 0 & 0 & M^{\prime} c^{\prime}\end{array}\right)$

respectively, where $\eta=\frac{a_{0}}{\sqrt{2}}, c=\frac{a_{0}}{4}$ and $c^{\prime}=\frac{a_{0}}{2 \sqrt{3}}$, and $a_{0}$ is a theoretically estimated cubic lattice constant of a diamond crystal, $3.571 \AA$. $N$ and $M\left(M^{\prime}\right)$ specify the periodicities in the surface-parallel and surface-normal directions of the $\mathrm{C}(001)[\mathrm{C}(111)]$ supercells $(N=4, M=28$ and $\left.M^{\prime}=30\right)$, respectively.
[10] D. Vanderbilt, Phys. Rev. B 41, 7892 (1990).

[11] K. Laasonen, A. Pasquarello, R. Car, C. Lee, and D. Vanderbilt, Phys. Rev. B 47, 10142 (1993).

[12] N. Troullier and J. L. Martins, Phys. Rev. B 43, 1993 (1991)

[13] Although the formation energy depends on the chemical potentials of $\mathrm{C}, \mathrm{P}$ and $\mathrm{H}$, the difference in the formation energies as defined in the text does not, since the chemical-potential terms are cancelled out when taking the difference between the formation energies for $\mathrm{P}$ in different positions.

[14] S. Hong and M. Y. Chou, Phys. Rev. B 57, 6262 (1998).

[15] The threshold is given by $\mu_{\mathrm{H}}^{0}=\frac{1}{4}\left\{E_{\mathrm{tot}}\left(\mathrm{CH}_{4}\right)-\mu_{\mathrm{C}}\right\}$, where $E_{\text {tot }}\left(\mathrm{CH}_{4}\right)$ is the total energy of a methane molecule and $\mu_{\mathrm{C}}$ is the total energy (per C atom) of a diamond crystal. Hong and Chou[14] added the contribution of zero-point energy of a $\mathrm{H}$ atom to $E_{\text {tot }}\left(\mathrm{CH}_{4}\right)$.

[16] H. Kato, S. Yamasaki, and H. Okushi, Diamond Relat. Mater. , in press.

[17] H. Kato, H. Futako, S. Yamasaki, and H. Okushi, Diamond Relat. Mater. 14, 340 (2005). 